\title{
Heisenberg-Kitaev models on hyperhoneycomb and stripy-honeycomb lattices: 3D-2D equivalence of ordered states and phase diagrams
}

\author{
Wilhelm G. F. Krüger (), Matthias Vojta, and Lukas Janssen (1) \\ Institut für Theoretische Physik and Würzburg-Dresden Cluster of Excellence ct.qmat, \\ Technische Universität Dresden, 01062 Dresden, Germany
}

(Received 22 July 2019; published 21 January 2020)

\begin{abstract}
We discuss magnetically ordered states, arising in Heisenberg-Kitaev and related spin models, on threedimensional (3D) harmonic honeycomb lattices. For large classes of ordered states, we show that they can be mapped onto two-dimensional (2D) counterparts on the honeycomb lattice, with the classical energetics being identical in the $2 \mathrm{D}$ and $3 \mathrm{D}$ cases. As an example, we determine the phase diagram of the classical nearest-neighbor Heisenberg-Kitaev model on the hyperhoneycomb lattice in a magnetic field: this displays rich and complex behavior akin to its 2D counterpart, with most phases and phase boundaries coinciding exactly. To make contact with the physics of the hyperhoneycomb iridate $\beta$ - $\mathrm{Li}_{2} \mathrm{IrO}_{3}$, we also include a symmetric off-diagonal $\Gamma$ interaction, discuss its 3D-2D mapping, and determine the relevant phase diagrams. In particular, we demonstrate explicitly the adiabatic equivalence of the spiral magnetic orders in $\alpha$ - and $\beta-\mathrm{Li}_{2} \mathrm{IrO}_{3}$. Our results pave the way to a systematic common understanding of 2D and 3D Kitaev materials.
\end{abstract}

DOI: 10.1103/PhysRevResearch.2.012021

In studies of quantum magnetism, materials with strong spin-orbit coupling have moved to center stage, as they promise to realize novel phases beyond those known for spinsymmetric Heisenberg models [1-3]. A paradigmatic example for nontrivial effects of spin-anisotropic interactions is $\mathrm{Ki}$ taev's celebrated honeycomb-lattice spin model, being exactly solvable and realizing a $\mathbb{Z}_{2}$ spin liquid of Majorana fermions [4]. In the search for materials realizations, compounds with $4 d$ and $5 d$ transition-metal ions arranged on layered honeycomb lattices have been proposed [1], such as $\mathrm{Na}_{2} \mathrm{IrO}_{3}$, $\alpha-\mathrm{Li}_{2} \mathrm{IrO}_{3}$, and $\alpha-\mathrm{RuCl}_{3}$ [5-9]. In these materials, the combined effect of spin-orbit coupling, Coulomb interaction, and exchange geometry generates $J_{\text {eff }}=1 / 2$ moments subject to a combination of exchange interactions, the most important ones being Kitaev, Heisenberg, and symmetric off-diagonal [10-12]. These two-dimensional (2D) Kitaev materials have generated tremendous interest, and the relevant extended $\mathrm{Ki}$ taev models have been shown to host both spin-liquid and symmetry-broken phases $[13,14]$.

Beyond two dimensions, it has been shown that the Kitaev model can also be exactly solved on particular threedimensional (3D) lattices with threefold coordination $[15,16]$, and the materials $\beta$ - $\mathrm{Li}_{2} \mathrm{IrO}_{3}[17,18]$ and $\gamma-\mathrm{Li}_{2} \mathrm{IrO}_{3}[19,20]$ were found to realize two of these lattices, the hyperhoneycomb and stripyhoneycomb lattices. In fact, these two are part of an infinite family of 3D lattices whose limiting case is the $2 \mathrm{D}$ honeycomb lattice-the harmonic-honeycomb series

Published by the American Physical Society under the terms of the Creative Commons Attribution 4.0 International license. Further distribution of this work must maintain attribution to the author(s) and the published article's title, journal citation, and DOI.
$[15,19,21]$. While $\mathrm{Na}_{2} \mathrm{IrO}_{3}$ and $\alpha-\mathrm{RuCl}_{3}$ display collinear zigzag order $[6,7,22]$ at low temperatures, the magnetic states of the $\mathrm{Li}_{2} \mathrm{IrO}_{3}$ polytypes involve noncollinear spin spirals. In an applied magnetic field, the spiral order in $\beta-\mathrm{Li}_{2} \mathrm{IrO}_{3}$ is rapidly suppressed, giving way to a zigzag state analogous to those of the planar honeycomb materials [23,24]. While a number of concrete studies of either $2 \mathrm{D}$ or $3 \mathrm{D}$ models have appeared, a common systematic understanding is lacking.

In this Rapid Communication, we consider magnetically ordered states on the 3D hyperhoneycomb and other harmonic honeycomb lattices and establish a remarkable correspondence to states on the 2D honeycomb lattice: For large classes of states, we demonstrate a precise mapping between 3D and $2 \mathrm{D}$, with the ground-state and magnon-mode energies, as well as other momentum-resolved observables, such as the dynamic spin structure factor, being identical in the semiclassical limit. As an example, we determine the phase diagram of the classical nearest-neighbor Heisenberg-Kitaev model on the hyperhoneycomb lattice in a magnetic field: We show that this is almost identical to that of the same model on the honeycomb lattice [25], with the exception of a single field-induced phase that is of genuine $3 \mathrm{D}$ character and thus escapes the 3D-2D mapping. In order to model $\beta-\mathrm{Li}_{2} \mathrm{IrO}_{3}$, we include symmetric off-diagonal $\Gamma$ interactions on the hyperhoneycomb lattice, which can induce incommensurate spiral phases, and we discuss their exact 3D-2D mapping. Finally, we consider effects beyond the classical limit and compare quantum corrections to the magnetization between the $3 \mathrm{D}$ and 2D cases.

a. Mapping from 3D to 2D: general. The hyperhoneycomb lattice is a tricoordinated $3 \mathrm{D}$ lattice that can be classified as face-centered orthorhombic with a four-site atomic unit cell [26,27]. In Fig. 1(a), we show the spin configuration of a so-called skew-zigzag antiferromagnetic state on this lattice, 
(a)

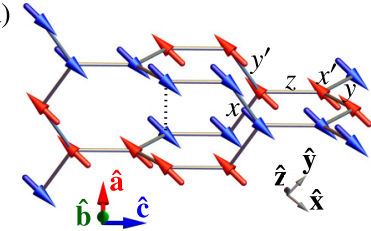

(c)

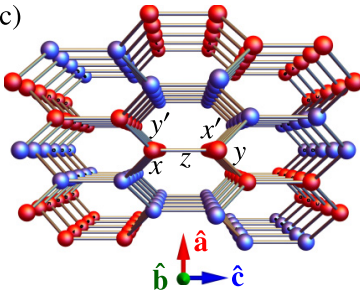

(b)

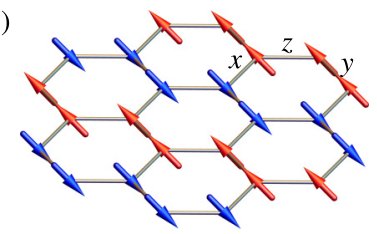

(d)

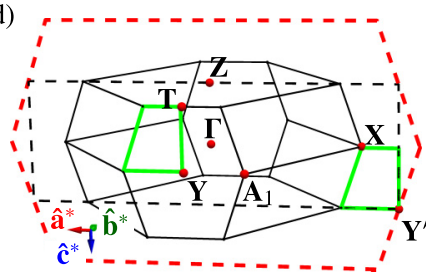

FIG. 1. (a) Hyperhoneycomb lattice with magnetic skew-zigzag state and (b) honeycomb lattice with zigzag state; both are equivalent as explained in the text. (c) Hyperhoneycomb lattice viewed from the crystallographic $\hat{\mathbf{b}}$ direction, illustrating its projection onto an elongated honeycomb lattice. Colored balls indicate spin directions. (d) Brillouin zones of the hyperhoneycomb lattice (black) and the elongated honeycomb lattice (red dashed). 3D states with ordering wave vectors in the $a c$ plane can be transformed into equivalent $2 \mathrm{D}$ states on the honeycomb lattice. The quarters of the front hexagon (such as the green quadrangle) can be shifted with reciprocal lattice vectors to the $a c$ plane and form, together with the $a c$-plane cut of the first Brillouin zone, a rectangle (black dashed). The latter becomes the 2D Brillouin zone of the elongated honeycomb lattice if a four-site unit cell is chosen. All high-symmetry points shown are quasi-2D.

while Fig. 1(b) displays a corresponding zigzag state on the $2 \mathrm{D}$ honeycomb lattice. These two states are equivalent in the following sense: projecting the hyperhoneycomb lattice onto its $a c$ plane results in an elongated honeycomb lattice, Fig. 1(c), and this projection transforms the skew-zigzag state from panel (a) into the zigzag state of panel (b). Importantly, their classical-limit energies are identical, because in both cases each site faces neighbors with identical alignment. This projective equivalence, which is the central result of this Rapid Communication, applies to all 3D ordered states where sites separated by the lattice vector $\mathbf{b}$ [dashed line in Fig. 1(a)] are magnetically equivalent. As we will see below, this includes large classes of 3D magnetic states, which we will dub "quasi-2D."

This 3D-2D equivalence holds despite the fact that the symmetry groups on the two lattices are obviously different. In particular, the number of sites in the primitive unit cell on the hyperhoneycomb lattice is four, and thus twice its value on the honeycomb lattice. It is thus possible to construct states (e.g., skew-zigzag states) that do not break the translation symmetry on the hyperhoneycomb lattice, although their 2D projections break the honeycomb translation symmetry.

Further insight is gained in reciprocal space. All highsymmetry points displayed in Fig. 1(d) have a vanishing component along the direction of the reciprocal lattice vector b* (up to reciprocal-lattice translations). States with ordering wave vectors at these high-symmetry points thus exhibit no modulation along the $\mathbf{b}$ axis in real space and are quasi2D: Their projection onto the $a c$ plane yields states on the honeycomb lattice with the exact same classical energy. This applies to all ordered phases in the nearest-neighbor Heisenberg-Kitaev (HK) model in zero field. Upon the inclusion of other symmetry-allowed interactions, as well as in a magnetic field, Kitaev systems also stabilize multi-Q and incommensurate states. We will show that even such more exotic states, including the counterrotating spiral states that are realized in the different $\mathrm{Li}_{2} \mathrm{IrO}_{3}$ polytypes, are quasi2D. Moreover, the 3D-2D mapping discussed here for the hyperhoneycomb lattice can be extended to the full harmonic honeycomb series, for details see the Supplemental Material [28]. Importantly, and in contrast to other families of lattices in different dimensions, all members of the harmonic honeycomb series have the same coordination number, which is a prerequisite for the 3D-2D equivalence to hold.

b. Heisenberg-Kitaev model in a magnetic field. To illustrate the power of the advertised mapping, we consider the spin- $S$ HK Hamiltonian $[11,13]$ in a uniform magnetic field $\mathbf{h}$,

$$
\mathcal{H}_{\mathrm{HK}}=J \sum_{\langle i j\rangle} \mathbf{S}_{i} \cdot \mathbf{S}_{j}+K \sum_{\langle i j\rangle_{\gamma}} S_{i}^{\gamma} S_{j}^{\gamma}-\mathbf{h} \cdot \sum_{i} \mathbf{S}_{i},
$$

where $\gamma \in\{x, y, z\}$ labels the three different types of bonds on the lattice. The 2D model on the honeycomb lattice has been studied intensely, see Refs. [3,32-34] for reviews. For nonzero field, the classical phase diagram (i.e., for $S \rightarrow \infty$ ) of this and related models has been determined [25,35-37], and the $S=1 / 2$ case has also been studied [38-47]. The 3D model on the hyperhoneycomb lattice in zero field has been considered in Ref. [26].

We have determined the phase diagram of the classical HK model in a magnetic field using a combination of high-field spin-wave theory and classical energy minimization. On the hyperhoneycomb lattice, the number of possible geometries of the magnetic unit cell drastically increases with its size. For reasons of numerical feasibility and consistency, we have restricted the numerical energy minimization in both the 2D and $3 \mathrm{D}$ cases to states with up to 12 sites in the magnetic unit cell, but have included all possible unit-cell geometries [28]. On the honeycomb lattice, our findings are consistent with the previous analyses $[25,48]$.

The result, comparing the hyperhoneycomb and honeycomb cases for a field along the $[111] \propto(\hat{\mathbf{x}}+\hat{\mathbf{y}}+\hat{\mathbf{z}}) / \sqrt{3}$ direction, is displayed in Fig. 2. The various phases are characterized in Ref. [28]. Remarkably, both phase diagrams agree quantitatively, with the exception of the 3D spiral phase, which appears only in the 3D case of panel (a). Inspecting the individual phases, we see that all phases except the 3D spiral have ordering wave vectors located in the $a c$ plane, such that 3D-2D mapping applies, whereas the 3D spiral phase has $\mathbf{Q}=\frac{1}{3} \mathbf{b}^{*}$, evading the mapping. The latter is hence a genuine 3D phase, consisting of spirals along the $\mathbf{b}$ direction in a 12-site magnetic unit cell, see Fig. 2(c).

We note that early work on the hyperhoneycomb-HK model in a magnetic field [49] missed the nontrivial fieldinduced phases found here. We have explicitly checked that our novel intermediate phases have lower energies than the canted skew-zigzag and skew-stripy states suggested in Ref. [49]. 

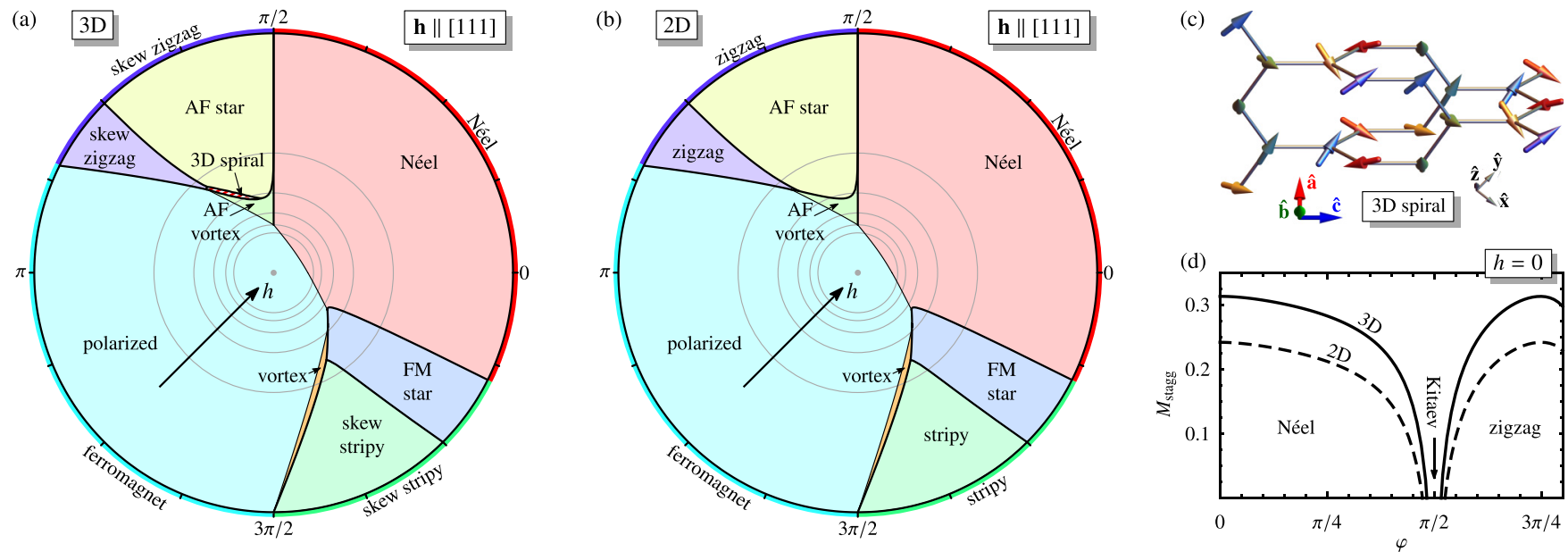

FIG. 2. Phase diagram of the classical HK model in a magnetic field $\mathbf{h}$ along the $[111] \propto(\hat{\mathbf{x}}+\hat{\mathbf{y}}+\hat{\mathbf{z}}) / \sqrt{3}$ direction for $T \rightarrow 0$, with $J=A \cos \varphi, K=2 A \sin \varphi$ [50], and the radial direction representing the field strength $h$, with $h /(A S)=1,2,3,4,5$ from outer to inner gray circles, on the (a) hyperhoneycomb lattice and (b) honeycomb lattice. The latter agrees with the previous analysis [25,35], with the exception of small regions for which the true ground state has incommensurate ordering wave vectors. Thick (thin) black lines denote first-order (second-order) phase transitions. Except for the 3D spiral phase, for which a representative spin configuration is shown in (c), all phases of the hyperhoneycomb lattice have a 2D analog with exactly the same ground-state energy. (d) Order parameters in Néel and zigzag phases from linear spin-wave theory for $S=1 / 2$ and zero field, comparing the hyperhoneycomb (solid) and honeycomb (dashed) lattices.

c. $\Gamma$ and other interactions. For actual Kitaev materials, it has been shown that, in addition to nearest-neighbor $\mathrm{Ki}$ taev and Heisenberg interactions, also symmetric off-diagonal interactions, commonly dubbed $\Gamma$ interactions, are important $[12,36,51-55]$. To model $\beta-\mathrm{Li}_{2} \mathrm{IrO}_{3}$, we hence consider the Hamiltonian

$$
\mathcal{H}_{\mathrm{HK} \pm \Gamma}=\sum_{\langle i j\rangle_{\gamma}}\left[J \mathbf{S}_{i} \cdot \mathbf{S}_{j}+K S_{i}^{\gamma} S_{j}^{\gamma} \pm \Gamma\left(S_{i}^{\alpha} S_{j}^{\beta}+S_{i}^{\beta} S_{j}^{\alpha}\right)\right],
$$

where $\alpha$ and $\beta$ label the two remaining directions on a $\gamma$ bond. On the hyperhoneycomb lattice, there are two inequivalent types of pairwise parallel $x(y)$ bonds, denoted as $x$ and $x^{\prime}$ ( $y$ and $y^{\prime}$ ), respectively, while all $z$ bonds are equivalent; see Fig. 1(a). We take the upper (lower) sign in front of the $\Gamma$ interaction on $x, y$, and $z\left(x^{\prime}\right.$ and $\left.y^{\prime}\right)$ bonds; this choice can be justified microscopically [52]. Applying the 3D-2D mapping to this model, which we dub $\mathrm{HK} \pm \Gamma$ model, we see that it corresponds to an unusual 2D model. Compared to the Heisenberg-Kitaev- $\Gamma$ (HK $\Gamma)$ model typically considered for 2D Kitaev materials, this has a supermodulation in the $\Gamma$ interaction. Remarkably, in the limit of vanishing $J$, the resulting $\mathrm{K} \pm \Gamma$ and $\mathrm{K} \Gamma$ models can be shown to be dual to each other by using a unitary transformation that rotates the spins on consecutive zigzag chains by $\pi / 2$ and $3 \pi / 2$, respectively, about the $z$ axis [28]. In the presence of sizable $\Gamma$ interactions, incommensurate states appear [12,52].

Despite these complications, the concept of the 3D-2D mapping continues to apply. We illustrate this in Fig. 3, where we show the classical phase diagrams of the $\mathrm{HK} \pm \Gamma$ model at zero field for the hyperhoneycomb and honeycomb lattices. These have been obtained via a combination of a LuttingerTisza analysis and a single-Q ansatz (as the finite-cluster minimization does not capture incommensurate states) [28]. Our result on the hyperhoneycomb lattice agrees with the previous work [52], except for a small region around the $\mathrm{AF}_{a b c}$ phase, for which the true ground state may be a multi- $\mathbf{Q}$ state that is beyond our ansatz. Again, the 3D and 2D phase diagrams agree quantitatively, with the exception of the $\overline{\mathrm{SP}}_{b^{ \pm}}$and $\mathrm{AF}_{a b c}$ phases, which appear only in panel (a) and are thus genuinely 3D. This result is particularly striking for the counterrotating spiral $\overline{\mathrm{SP}}_{a^{ \pm}}$phases, for which the ground state is incommensurate, but with an ordering wave vector $\mathbf{Q} \| \mathbf{a}^{*}$, i.e., within the $a c$ plane; cf. Figs. 3(c) and 3(d). The $\overline{\mathrm{SP}}_{a^{-}}$phase includes the ground state realized in $\beta-\mathrm{Li}_{2} \mathrm{IrO}_{3}[17,52,53]$, such that 3D-2D mapping directly applies to this material. For small $J$, the $\overline{\mathrm{SP}}_{a^{-}}$phase becomes commensurate with ordering wave vector $\mathbf{Q}=\frac{1}{3} \mathbf{a}^{*}[54,55]$. The 3D-2D equivalence, together with the above-mentioned duality between the $\mathrm{K} \pm \Gamma$ and $\mathrm{K} \Gamma$ models, maps this state to the coplanar $120^{\circ}$ state on the honeycomb lattice [12], which is indeed close to the observed magnetic order in the planar polytype $\alpha-\mathrm{Li}_{2} \mathrm{IrO}_{3}$ [8]. In fact, the mapping explains several characteristic common features observed in the different $\mathrm{Li}_{2} \mathrm{IrO}_{3}$ polytypes: (i) as a consequence of the structure of the duality transformation, the hyperhoneycomb-lattice state corresponding to the $120^{\circ}$ state consists of zigzag chains with coplanar spins, in agreement with the experimental findings in $\beta-\mathrm{Li}_{2} \mathrm{IrO}_{3}$ [17]. (ii) In the $120^{\circ}$ phase on the honeycomb lattice, the spins on the two sublattices rotate in opposite directions, which explains the emergence of counterrotating spirals on the hyperhoneycomb lattice. (iii) The duality between $\overline{\mathrm{SP}}_{a^{-}}$and $120^{\circ}$ states for small $J$ furthermore reveals why the angle between every second spin of a zigzag chain is close to $120^{\circ}$ in both $\alpha$ - and $\beta-\mathrm{Li}_{2} \mathrm{IrO}_{3}[8,17]$.

For completeness, we note that the $3 \mathrm{D}-2 \mathrm{D}$ mapping can be extended to interactions beyond nearest neighbors; for details see Ref. [28].

d. Beyond the classical limit. While the advertised qualitative 3D-2D mapping of ordered states is very general, their quantitative energetic equivalence only applies to the classical limit, $S \rightarrow \infty$. We have therefore studied quantum effects in a systematic $1 / S$ expansion using spin-wave theory. 

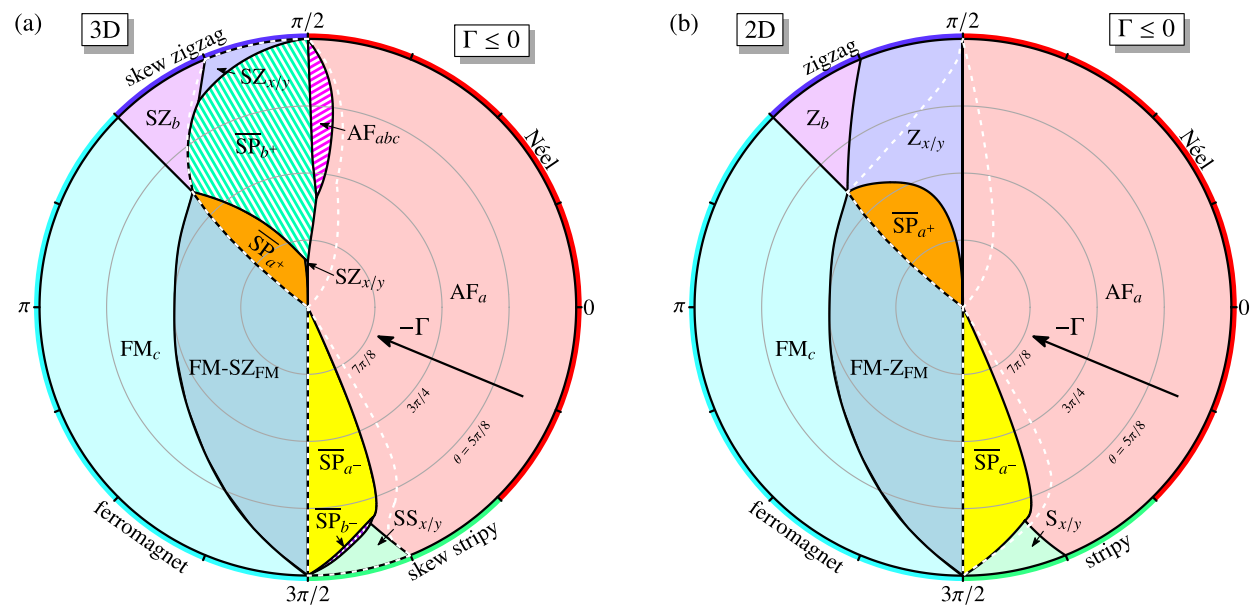

(c)

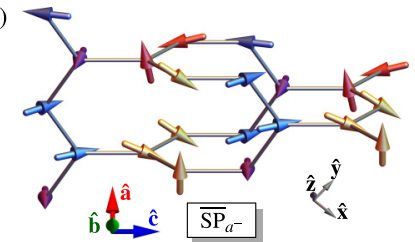

(d)

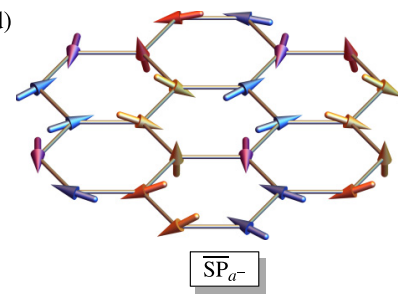

FIG. 3. Phase diagram of the classical HK $\pm \Gamma$ model for $T \rightarrow 0$, with $J=A \sin \theta \cos \varphi, K=A \sin \theta \sin \varphi$, and $\Gamma=A \cos \theta \leqslant 0$ [50] on the (a) hyperhoneycomb lattice [52] and (b) honeycomb lattice. Hatched regions in (a) denote genuine 3D phases with ordering wave vectors outside the $a c$ plane. The white dashed line indicates the regions in which the Luttinger-Tisza approach fails to satisfy the local length constraint and a single- $\mathbf{Q}$ ansatz has been employed instead [28]. The 3D-2D equivalence holds also for the incommensurate spiral phase $\overline{\mathrm{SP}}_{a^{-}}, \mathrm{relevant}$ for $\beta-\mathrm{Li}_{2} \mathrm{IrO}_{3}$, for which a representative spin configuration on the hyperhoneycomb lattice is plotted in (c), together with its projection onto the honeycomb lattice in (d).

A first remarkable insight is that the leading-order magnon spectra and the dynamic structure factors also follow the 3D-2D mapping, i.e., the magnon energies and weights on the different harmonic honeycomb lattices are identical for 3D wave vectors belonging to the $a c$ plane; this is demonstrated explicitly in Ref. [28].

In Fig. 2(d), we display the order parameters (i.e., staggered magnetizations) evaluated for $S=1 / 2$ in the Néel and zigzag phases of the HK model at zero field, comparing the hyperhoneycomb and honeycomb lattices. In the hyperhoneycomb case, the quantum corrections to the classical value $M_{\text {stagg }}=1 / 2$ are smaller, but the qualitative behavior of the order parameter is similar to those of the honeycomb lattice. In particular, in linear spin-wave theory, the critical couplings at which the order parameters vanish, indicating the transition to the Kitaev spin liquid phase, roughly agree. This suggests that the Kitaev spin liquid on the hyperhoneycomb lattice [56] covers a parameter range that is only slightly smaller than those of its honeycomb-lattice counterpart.

These results illustrate that the different phase space renders quantum fluctuations stronger in $2 \mathrm{D}$ compared to $3 \mathrm{D}$. As a result, phase boundaries will shift and spoil the exact 3D-2D equivalence for $S<\infty$. This also means that classical phases that are destroyed by quantum fluctuations in 2D possibly survive in the $3 \mathrm{D}$ case.

e. Summary. In this paper, we have established an exact correspondence between magnetically ordered spin states on the 3D harmonic honeycomb lattices and the 2D planar honeycomb lattice. This correspondence is quantitative in the classical limit and applies to large classes of ordered states.
The condition is that the respective 3D ordering wave vector lies in the $a c$ plane (up to reciprocal-lattice translations), which pertains to all high-symmetry points in the Brillouin zone. We have demonstrated this 3D-2D mapping for the hyperhoneycomb-lattice Heisenberg-Kitaev model in a magnetic field, where we found exact agreement with the 2D case, with the exception of one intermediate phase which is of genuine $3 \mathrm{D}$ character.

The hyperhoneycomb material $\beta-\mathrm{Li}_{2} \mathrm{IrO}_{3}$ orders in a counterrotating-spiral state at low temperatures $[17,18]$. Our 3D-2D mapping, together with a duality transformation, demonstrates that this state can be understood as an adiabatic deformation of the $120^{\circ}$ state on the honeycomb lattice, which is close to the magnetic order in $\alpha-\mathrm{Li}_{2} \mathrm{IrO}_{3}$ [8]. This result establishes the equivalence of the experimentally observed spiral states in the different $\mathrm{Li}_{2} \mathrm{IrO}_{3}$ polytypes. $\beta-\mathrm{Li}_{2} \mathrm{IrO}_{3}$ exhibits a nontrivial behavior in a finite magnetic field $[23,24,54]$. The 3D-2D equivalence suggests that similarly interesting in-field effects may occur also in $\alpha$ - and $\gamma-\mathrm{Li}_{2} \mathrm{IrO}_{3}$ $[57,58]$. Together, our work paves the way to a unified understanding of the magnetism in 3D and 2D Kitaev materials and opens novel perspectives for dimensional diversification.

Acknowledgments. We thank N. B. Perkins for enlightening discussions and E. C. Andrade for collaboration on earlier related work. This research was supported by the DFG through SFB 1143 (project id 247310070), the Würzburg-Dresden Cluster of Excellence on Complexity and Topology in Quantum Matter - ct.qmat (EXC 2147, project id 390858490), and the Emmy Noether program (JA2306/4-1, project id 411750675).
[1] G. Jackeli and G. Khaliullin, Phys. Rev. Lett. 102, 017205 (2009).
[2] Z. Nussinov and J. van den Brink, Rev. Mod. Phys. 87, 1 (2015). 
[3] J. G. Rau, E. K.-H. Lee, and H.-Y. Kee, Annu. Rev. Condens. Matter Phys. 7, 195 (2016).

[4] A. Kitaev, Ann. Phys. 321, 2 (2006).

[5] Y. Singh, S. Manni, J. Reuther, T. Berlijn, R. Thomale, W. $\mathrm{Ku}, \mathrm{S}$. Trebst, and P. Gegenwart, Phys. Rev. Lett. 108, 127203 (2012).

[6] S. K. Choi, R. Coldea, A. N. Kolmogorov, T. Lancaster, I. I. Mazin, S. J. Blundell, P. G. Radaelli, Y. Singh, P. Gegenwart, K. R. Choi, S.-W. Cheong, P. J. Baker, C. Stock, and J. Taylor, Phys. Rev. Lett. 108, 127204 (2012).

[7] J. A. Sears, M. Songvilay, K. W. Plumb, J. P. Clancy, Y. Qiu, Y. Zhao, D. Parshall, and Y.-J. Kim, Phys. Rev. B 91, 144420 (2015).

[8] S. C. Williams, R. D. Johnson, F. Freund, S. Choi, A. Jesche, I. Kimchi, S. Manni, A. Bombardi, P. Manuel, P. Gegenwart, and R. Coldea, Phys. Rev. B 93, 195158 (2016).

[9] S. M. Winter, A. A. Tsirlin, M. Daghofer, J. van den Brink, Y. Singh, P. Gegenwart, and R. Valentí, J. Phys.: Condens. Matter 29, 493002 (2017).

[10] A. Shitade, H. Katsura, J. Kuneš, X.-L. Qi, S.-C. Zhang, and N. Nagaosa, Phys. Rev. Lett. 102, 256403 (2009).

[11] J. Chaloupka, G. Jackeli, and G. Khaliullin, Phys. Rev. Lett. 105, 027204 (2010).

[12] J. G. Rau, E. K.-H. Lee, and H.-Y. Kee, Phys. Rev. Lett. 112, 077204 (2014).

[13] J. Chaloupka, G. Jackeli, and G. Khaliullin, Phys. Rev. Lett. 110, 097204 (2013)

[14] C. C. Price and N. B. Perkins, Phys. Rev. Lett. 109, 187201 (2012).

[15] I. Kimchi, J. G. Analytis, and A. Vishwanath, Phys. Rev. B 90, 205126 (2014).

[16] K. O’Brien, M. Hermanns, and S. Trebst, Phys. Rev. B 93, 085101 (2016).

[17] A. Biffin, R. D. Johnson, S. Choi, F. Freund, S. Manni, A. Bombardi, P. Manuel, P. Gegenwart, and R. Coldea, Phys. Rev. B 90, 205116 (2014).

[18] T. Takayama, A. Kato, R. Dinnebier, J. Nuss, H. Kono, L. S. I. Veiga, G. Fabbris, D. Haskel, and H. Takagi, Phys. Rev. Lett. 114, 077202 (2015).

[19] K. A. Modic et al., Nat. Commun. 5, 4203 (2014).

[20] A. Biffin, R. D. Johnson, I. Kimchi, R. Morris, A. Bombardi, J. G. Analytis, A. Vishwanath, and R. Coldea, Phys. Rev. Lett. 113, 197201 (2014).

[21] I. Kimchi, R. Coldea, and A. Vishwanath, Phys. Rev. B 91, 245134 (2015).

[22] F. Ye, S. Chi, H. Cao, B. C. Chakoumakos, J. A. FernandezBaca, R. Custelcean, T. F. Qi, O. B. Korneta, and G. Cao, Phys. Rev. B 85, 180403(R) (2012).

[23] A. Ruiz, A. Frano, N. P. Breznay, I. Kimchi, T. Helm, I. Oswald, J. Y. Chan, R. J. Birgeneau, Z. Islam, and J. G. Analytis, Nat. Commun. 8, 961 (2017).

[24] M. Majumder, F. Freund, T. Dey, M. Prinz-Zwick, N. Büttgen, Y. Skourski, A. Jesche, A. A. Tsirlin, and P. Gegenwart, Phys. Rev. Mater. 3, 074408 (2019).

[25] L. Janssen, E. C. Andrade, and M. Vojta, Phys. Rev. Lett. 117, 277202 (2016).

[26] E. K.-H. Lee, R. Schaffer, S. Bhattacharjee, and Y. B. Kim, Phys. Rev. B 89, 045117 (2014).
[27] M. Hermanns and S. Trebst, Phys. Rev. B 89, 235102 (2014).

[28] See Supplemental Material at http://link.aps.org/supplemental/ 10.1103/PhysRevResearch.2.012021, which also contains Refs. [29-31], for a generalization of the 3D-2D mapping on the harmonic honeycomb lattice series including interactions beyond the $\mathrm{HK} \pm \Gamma$ models, details of the different methods used, characterizations of the various phases found, and a discussion of quantum corrections in linear spin-wave theory.

[29] J. M. Luttinger and L. Tisza, Phys. Rev. 70, 954 (1946).

[30] D. H. Lyons and T. A. Kaplan, Phys. Rev. 120, 1580 (1960).

[31] J. Chaloupka and G. Khaliullin, Phys. Rev. B 92, 024413 (2015).

[32] S. Trebst, arXiv:1701.07056.

[33] M. Hermanns, I. Kimchi, and J. Knolle, Annu. Rev. Condens. Matter Phys. 9, 17 (2018).

[34] L. Janssen and M. Vojta, J. Phys.: Condens. Matter 31, 423002 (2019).

[35] G.-W. Chern, Y. Sizyuk, C. Price, and N. B. Perkins, Phys. Rev. B 95, 144427 (2017).

[36] L. Janssen, E. C. Andrade, and M. Vojta, Phys. Rev. B 96, 064430 (2017).

[37] L. E. Chern, R. Kaneko, H.-Y. Lee, and Y. B. Kim, arXiv:1905.11408.

[38] H.-C. Jiang, Z.-C. Gu, X.-L. Qi, and S. Trebst, Phys. Rev. B 83, 245104 (2011).

[39] M. Gohlke, R. Moessner, and F. Pollmann, Phys. Rev. B 98, 014418 (2018).

[40] Z. Zhu, I. Kimchi, D. N. Sheng, and L. Fu, Phys. Rev. B 97, 241110(R) (2018).

[41] D. C. Ronquillo, A. Vengal, and N. Trivedi, Phys. Rev. B 99, 140413(R) (2019).

[42] H.-C. Jiang, C.-Y. Wang, B. Huang, and Y.-M. Lu, arXiv:1809.08247.

[43] L. Zou and Y.-C. He, arXiv:1809.09091.

[44] C. Hickey and S. Trebst, Nat. Commun. 10, 530 (2019).

[45] N. D. Patel and N. Trivedi, Proc. Natl. Acad. Sci. USA 116, 12199 (2019).

[46] J. Nasu, Y. Kato, Y. Kamiya, and Y. Motome, Phys. Rev. B 98, 060416(R) (2018).

[47] S. Liang, M.-H. Jiang, W. Chen, J.-X. Li, and Q.-H. Wang, Phys. Rev. B 98, 054433 (2018).

[48] Our method finds the correct single- or multi-Q ground state up to small regions in the phase diagram which exhibit incommensurate multi-Q order, only detectable by large-scale Monte Carlo simulations [35].

[49] S.-B. Lee, E. K.-H. Lee, A. Paramekanti, and Y. B. Kim, Phys. Rev. B 89, 014424 (2014).

[50] We follow the parametrization of Heisenberg and Kitaev couplings in terms of the angle $\varphi$ as used in the literature, which differ by a factor of two for the $\mathrm{HK}$ and $\mathrm{HK} \pm \Gamma$ models: $K / J=2 \tan \varphi(\mathrm{HK})$ and $K / J=\tan \varphi(\mathrm{HK} \pm \Gamma)$, respectively. $A>0$ denotes an overall energy scale.

[51] S. M. Winter, Y. Li, H. O. Jeschke, and R. Valentí, Phys. Rev. B 93, 214431 (2016)

[52] E. K.-H. Lee and Y. B. Kim, Phys. Rev. B 91, 064407 (2015).

[53] E. K.-H. Lee, J. G. Rau, and Y. B. Kim, Phys. Rev. B 93, 184420 (2016). 
[54] S. Ducatman, I. Rousochatzakis, and N. B. Perkins, Phys. Rev. B 97, 125125 (2018).

[55] I. Rousochatzakis and N. B. Perkins, Phys. Rev. B 97, 174423 (2018).

[56] S. Mandal and N. Surendran, Phys. Rev. B 79, 024426 (2009).
[57] S. Choi, S. Manni, J. Singleton, C. V. Topping, T. Lancaster, S. J. Blundell, D. T. Adroja, V. Zapf, P. Gegenwart, and R. Coldea, Phys. Rev. B 99, 054426 (2019).

[58] K. A. Modic, B. J. Ramshaw, J. B. Betts, N. P. Breznay, J. G. Analytis, R. D. McDonald, and A. Shekhter, Nat. Commun. 8, 180 (2017). 